\title{
THE SMALL SATELLITES OF THE SOLAR SYSTEM: PRIORITIES FOR THE DECADAL STUDY \\ July 15, 2020
}

Bonnie J. Buratti (Jet Propulsion Laboratory, California Institute of Technology, 818-468-1401, bonnie.j.buratti@jpl.nasa.gov), , Erik Asphaug (University of Arizona), James Bauer (University of Maryland), Julie Bellerose (JPL), David Blewett (JHU/APL), William Bottke (Southwest Research Institute), Daniel Britt (Univ. of Central Florida), Julie Castillo-Rogez (JPL/Caltech), Tilmann Denk (DLR), Nader Haghighipour (IFA/Univ. of Hawaii), Jian-Yang Li (Planetary Sciences Institute), David Nesvorny (Southwest Research Institute), Andrew Rivkin (JHU/APL), Daniel Scheeres (Univ. of Colorado), Mark V. Sykes (Planetary Science Institute), Peter Thomas (Cornell Univ.), Anne Verbiscer (Univ. of Virginia), Faith Vilas (Planetary Science Institute), Hajime Yano (JAXA/ISAS \& JSPEC), Richard Cartwright (SETI Institute), Timothy Holt (University of Southern Queensland)

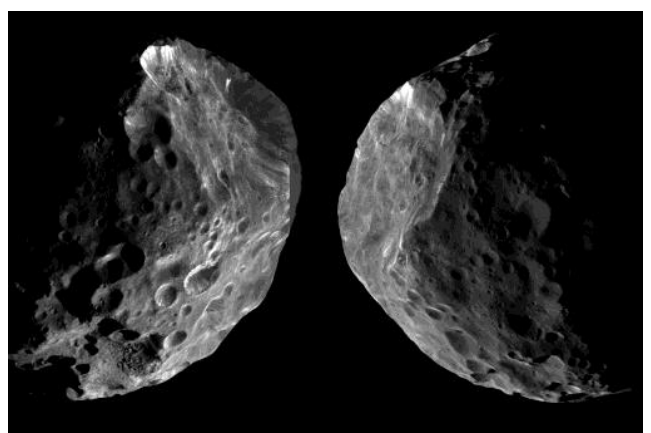

Phoebe (NASA PIA Image 18411)

\section{Abstract}

The small satellites are a diverse group of objects offering insights into the early formation of the Solar System and its collisional history. They can be subdivided into several groups: small inner moons, outer irregular moons, Martian moons, moons of asteroids, and moons of Centaurs/KBOs/TNOs. Many of these objects, especially the irregulars or their progenitors, are thought to be captured asteroids or Kuiper Belt Objects: a spacecraft mission that included them as targets would thus yield information on an object that came from elsewhere in the Solar System. Objects originating from the same reservoir as the irregular satellites may have brought both pre-biotic material and volatiles such as water to the inner Solar System. Flagship and New Frontiers class missions should plan trajectories and arrival times to capture spectroscopic and imaging observations of small satellites. It is also important to support ground-based and Earthorbiting observations of these objects, laboratory measurements of candidate surface materials, and curation facilities for organic materials and ice- and gas-based materials. We place a high priority on a robust participating scientist program for JAXA's MMX mission to Phobos and Deimos, as a similar mission was our highest priority New Frontiers class mission in the last Decadal Survey. A sample returned by this mission would include material from a primitive object and possibly the Martian surface.

Scientific priorities for moons of asteroids and KBOs are covered in white papers for those objects 


\section{The small satellites: An overview}

The small satellites are a diverse group offering insights into the formation of planetary systems, the primordial conditions and current architecture of gaseous planet-systems, and the migration of small bodies within the Solar System. Most of the outer moons are likely remnants of catastrophic collisions, and their progenitor objects are thought to have been asteroids or Kuiper Belt Objects (KBOs) that were captured around the giant planets by a dynamical mechanism (see reviews by Jewitt and Haghighipour 2007; Nicholson et al. 2008). Among the inner moons, some orbit within the gaps of Saturn's rings and around their edges (Table 1). Many of the small moons show evidence of violent pasts: one is in chaotic rotation (Hyperion) and others are heavily cratered. They are compositionally diverse, ranging from low-albedo C-type objects such as Phoebe to bright icy objects such as the inner satellites of Saturn. Captured bodies offer windows into the physical conditions of remote regions of the Solar System. The scope of this paper includes both distant captured (so-called "irregular") satellites and small inner satellites. All of these objects are irregular in shape and are $\sim 200 \mathrm{~km}$ or smaller in radius.

Table 1 - A summary of the small satellites of the Solar System planets (including Pluto)

\begin{tabular}{|c|c|c|c|c|c|}
\hline Planet & $\begin{array}{l}\text { Satellite or } \\
\text { group }\end{array}$ & \# & $\begin{array}{l}\text { Distance from primary } \\
(\mathbf{k m})\end{array}$ & $\begin{array}{l}\text { Radii } \\
(\mathbf{k m})\end{array}$ & Comments \\
\hline \multirow[t]{2}{*}{ Mars } & Phobos & & 9376 & 11.1 & \multirow{2}{*}{$\begin{array}{l}\text { Captured C or D asteroids; each one } \\
\text { is unique }\end{array}$} \\
\hline & Deimos & & 23458 & 6.2 & \\
\hline \multirow[t]{2}{*}{ Jupiter } & Inner moons & 4 & $128,000-222,000$ & $8-83$ & Amalthea largest \\
\hline & Outer moons & 71 & $7,284,000-28,347,000$ & $0.5-85$ & Most retrograde; breakup-families \\
\hline \multirow[t]{4}{*}{ Saturn } & Inner moons & 15 & $133,580-377,444$ & $1-89$ & "Shepherds", coorbitals, Lagrangians \\
\hline & Hyperion & & $1,500,993$ & 135 & Chaotic rotation \\
\hline & Phoebe & & $12,947,918$ & 107 & Captured KBO? \\
\hline & Outer moons & 57 & $11,384,000-26,676,000$ & $2-20$ & Many retrograde \\
\hline \multirow[t]{2}{*}{ Uranus } & Inner moons & 13 & $50,000-98,000$ & $9-81$ & Prograde \\
\hline & Outer moons & 9 & $4,282,900-20,430,000$ & $9-78$ & Retrograde (except one) \\
\hline \multirow[t]{3}{*}{ Neptune } & Inner moons & 6 & $48,227-117,646$ & $33-210$ & Prograde \\
\hline & Nereid & & $5,513,818$ & 170 & \\
\hline & Outer moons & 5 & $16,681,000-50,258,000$ & $9-31$ & 3 retrograde \\
\hline Pluto & Small moons & 4 & $42,393-64,698$ & $10-30$ & May be remnants of Charon \\
\hline
\end{tabular}

Source: JPL Horizons

Because the distantly orbiting irregular satellites exhibit collision probabilities typically four orders of magnitude higher than those found among main belt asteroids (Bottke et al. 2010), they are laboratories for collisional processes and formation scenarios for the outer planets. Nesvorny et al. $(2007 ; 2014)$ argued that outer irregular satellite capture may have taken place during giant planet close encounters within the Nice model framework (Tsiganis et al. 2005; Morbidelli et al., 2005). But the satellites' shallow Size-Frequency Distribution (SFD), which is markedly different from that of the Trojans, either argues against the Nice model or it indicates these bodies are the most collisionally-processed objects in the Solar System. The reservoirs from which the outer irregular satellites were captured may now be largely empty due to early scattering.

The small outer satellites also exhibit an ecological link to the rest of the Solar System. The dust - and larger pieces - from these collisions may provide material to alter the surfaces of major satellites such as Callisto, Iapetus, and the Uranian satellites (Buratti et al. 2005; Bottke et al., 
2010; Cartwright et al., 2018). The inner moons may provide material to the ring systems of the giant planets, and they in turn are coated with ring material. Both prebiotic compounds - the elusive dark material of the outer Solar System - and volatiles are brought into the inner solar system by comets, which share common origins with many of the small satellites. Finally, Pluto's moon-system may have dynamical origins analogous to the Earth's Moon (Canup and Asphaug, 2001). Some of the satellites orbit at such great distances from their primaries that they seem to be the "Oort clouds" of the giant planets. Perhaps the two populations even had the same source location, namely the primordial trans-planetary disk that was once located just beyond the orbits of the giant planets?

Although a dedicated future mission to a specific satellite is currently justified only for Phobos and Deimos, it is important to design any upcoming missions to capture opportunities to study these objects. An example of this planning was afforded by Cassini's flyby of Phoebe, which gave the first close view of what was probably once a KBO (Johnson and Lunine, 2005), and the first detailed dynamical study of Saturn's outer irregular moons (Denk and Mottola, 2019).

Figure 1. Some of the small, inner satellites of Saturn.

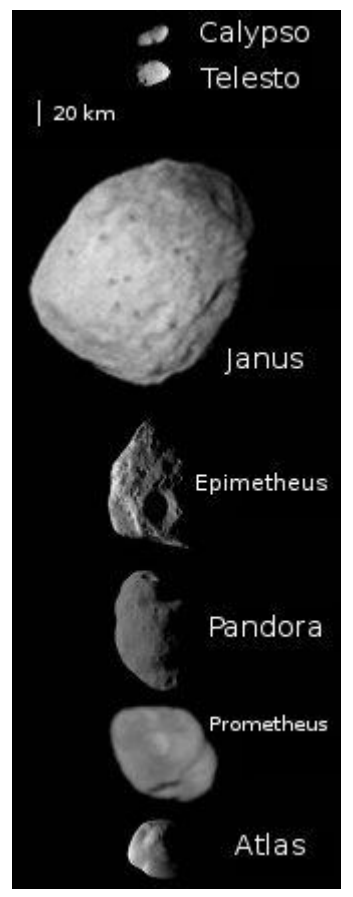

\section{Top-Level Scientific Questions}

The driving questions regarding small satellite research and missions include:

- What is the compositional and dynamical relationship between the small satellites and other bodies in the Solar System, including the outer main asteroid belt, Hildas, Jupiter and Neptune Trojans, KBOs (including the scattered disk), Centaurs, comets (including those from the Oort cloud), and the main satellite systems? Why do some small satellites appear to resemble P/D-type asteroids and others resemble C-type asteroids (e.g., Phoebe)? Is this difference intrinsic, due to evolutionary processes, or to some combination of the two?

- What determines the densities of the satellites? Specifically, why is the density of many so low? Are they rubble piles of loosely accreted material or material of a satellite that was broken apart and reaccreted?

- What mechanism captured the outer irregular satellites (or their progenitor objects), and how does this mechanism constrain planet formation processes in the outer Solar System? Were they captured from accretion regions near the giant planets during planet formation, or are they refugees from a massive primordial disk of comets that may have existed beyond the orbits of Uranus/Neptune? Can outer irregular satellites be used to verify or rule out the predictions made by Solar System formation scenarios?

- What are the interior composition and structure of Phobos and Deimos? Is there a dust ring associated with them, and can it reveal their composition?

- How has the high number of collisions shaped the outer irregular satellites (e.g., can we use 
these bodies to probe the nature of icy planetesimals?) Are there double-objects (binaries or contact-binaries), as thought to be common among the KBOs?

- How have the outer small satellites bombarded the main satellite systems? What is their role in the chronology of the main satellites as derived through crater counts?

- What is the relationship between the inner moons of Saturn, Uranus, Neptune and their rings? Are they compositionally related, and do the moons determine the morphology of the rings?

\section{Required Research and Research Facilities}

To address the above questions, a better understanding of the moons' composition, colors, albedos, dynamical states (rotation periods and pole positions), phase curves, sizes, and orbits is needed. Because of their distance and small size, the study of these moons has been largely done through broadband photometric analyses in the visible and near-infrared, leading to classifications based on colors. Advancements in our knowledge of their composition, including identification of specific volatiles, organics, and minerals, will be made through the acquisition of near-infrared spectra. Currently only $10-\mathrm{m}$ class telescopes are sufficiently equipped to provide these spectra, though additional observing time at facilities such as Keck would be helpful. Facilities including 10-m class telescopes and larger (e.g. TMT) need to be equipped with proper instrumentation for modest resolution spectroscopy and have dedicated time for planetary observations so that a statistically significant number of satellites can be thoroughly studied. It is especially important to continue to discover more outer satellites, to increase the pool of mission targets.

Observations with ALMA will provide size measurements and albedos for all known outer irregular satellites, and JWST will provide thermal infrared measurements, following on the advances made by Spitzer. NEOWISE has observed several of the largest irregulars of Jupiter and Saturn at 3-25 $\mu \mathrm{m}$. Especially valuable are observing campaigns of spacecraft targets to understand the rotational phase at the time of encounter, dynamics, albedo, and over-all context for planned mission measurements.

Also key are focused laboratory studies. Perhaps the most serious drawback to the identification of specific compounds on these moons (and other objects) is the lack of laboratory comparison spectra. NASA should support laboratory work to gather the spectra of volatiles, organics, and minerals at the temperatures appropriate to the outer Solar System. The effects of grain size, temperature, and viewing geometry should be studied. In addition, laboratory work on the mechanical properties of ices should also be supported. Support should be made available to have optical constants and other data online and easily accessible to investigators.

In advance of a sample return from MMX and other small body missions such as a comet surface sample return, NASA should provide investment in analytical facilities and training for the next generation of planetary sample scientists in the areas of organic materials and ice- and gas-based materials, as the Strategic Investments in Instrumentation and Facilities for Extraterrestrial Sample Curation and Analysis from the National Academies of Sciences, Engineering, and 
Medicine (2019) underlined. The impending return of samples from OSIRIS-REx and Hayabusa 2 underscores the urgency of the situation.

Results from campaigns by Cassini scientists to closely study the ring moons and outer small moons of Saturn have shown how mission plans that are optimized to observe small moons advance and complement Earth-based and laboratory studies (Denk and Mottola, 2019; Buratti et al., 2019; Tiscareno et al., 2019; Thomas and Helfenstein, 2020). The latter three papers have largely answered the last question of section II for Saturn. Denk and Mottola's systematic photometric survey of 25 outer irregular moons was the first of its kind and showed that the outer moons of Saturn are probably less dense than asteroids. Several objects (with Kiviuq being the best candidate) may be contact-binary or binary moons, suggesting they may be similar in morphology and origin to the small KBO 486958 Arrokoth, a remnant body that appears to be the conglomeration of smaller planetesimals (Stern et al., 2019).

\section{Technology Needs}

The needed technology developments for missions to small satellites include propulsion technologies, telecommunication technologies, sensing technologies, guidance navigation and control technologies (GN\&C), sampling technologies, and autonomy technology. These technologies are applicable across the spectrum of robotic mission categories, including missions to NEOs, asteroids, dwarf planets, Centaurs and KBOs as well as small irregular satellites.

\section{Major Mission Priorities}

Although studies of small satellites are not yet mature enough to warrant a dedicated Flagship Class Mission over the next decade, they are arguably as interesting as the Trojan asteroids and thus could potentially warrant their own New Frontiers mission in the follow-up to this Decadal Survey. In addition, a New Frontiers sample return mission to Phobos and Deimos is high priority, as JAXA has realized in its formulation of the MMX mission. One of our main messages is that the small satellites are superb secondary targets for big missions en route to other locations. The design of instruments and spacecraft should encompass the goals for small satellites. Any Mars mission should include the study of Phobos and Deimos. Whenever possible, spacecraft trajectories, arrival times, and instrument turn-on should be planned to capture the following measurements:

A. Flagship missions: A mission to the Saturnian or Jovian systems, as well as a possible Ice Giants flagship mission, should include observations during orbit insertion of one or more of the outermost satellites, as well as continued data-gathering throughout the mission of them and the small inner moons. The required observations include visible and IR spectroscopy to determine the moons' composition, and imaging to determine size, dynamics, crater frequency, and geologic history. Another important area is the dynamical interaction between the small inner satellites, ring systems, and the major satellites. Propellers, wakes, and gaps in the rings are all defined by small satellites orbiting in the ring regions: wherever possible high resolution imaging and spectroscopy of these objects should be planned. Europa Clipper should obtain observations of Amalthea and the other inner satellites if at all possible. 
B. New Frontiers Missions: A mission to Neptune (and Triton, if Trident or a similar mission is selected) or Uranus should include imaging and visible and infrared spectroscopic observations of outer satellites (with approaches as close as possible especially to the largest objects Nereid or Sycorax and possibly to Mab to understand its strange perturbed state) and of the inner moons (such as Proteus or Puck). These satellites will provide detailed views of the collisional environment in the outer Solar System, and compositional determinations will reveal kinships with KBOs, outer Main Belt asteroids, and the moons of the gas giants. Detailed studies of the small moons of Pluto by New Horizons reinforced the hypothesis that the small moons formed in the aftermath of a collision that produced the Pluto-Charon binary (Weaver et al., 2016). Although sample returns from Phobos and Deimos were proposed as Discovery Missions (Britt et al., 2003; Pieters et al., 2009) cost considerations currently place these missions in the New Frontiers class. These moons offer a window to study primitive materials that accreted in the outer part of the main asteroid belt, outside the terrestrial planet-forming zone. They are also deep in the Martian gravity well and thus have been accumulating ejecta from impacts on Mars. It has been suggested that up to $10 \%$ of Deimos' regolith may be Martian material (Britt, 2003; although Gladman (2007) has criticized this notion). Deimos is energetically more accessible than Phobos and has a remarkably smooth surface, rendering it "safe" for sampling operations.

A sample return mission to Phobos and Deimos was our only high priority, dedicated small moon mission for the last Decadal Survey. This mission would bring back to Earth primitive material as well as possible Martian material from the Noachian period since that is the period of maximum impact flux and of large basin formation. The main goals of the sample analysis would be to: study the geochemistry in the region of the outer asteroid belt and Jupiter; study prebiotic material in the Solar System; search for isotopic biomarkers on early Mars; determine the compositional diversity and history of the Martian crust, including dating the era of heavy bombardment. Martian ejecta accreted on Deimos would probably include crustal and upper mantle material from the period of early differentiation as well as later samples when Mars had a more dense atmosphere and possible surface water. Another goal of a mission to these moons mission would be to study the dust environment around the satellites and the possibility of a dust torus associated with the moons (Soter, 1971; Dubinin et al., 1990; Baumgärtel et al., 1996; Showalter, et al., 2006).

As JAXA has recently proposed the Martian Moons eXploration (MMX) mission, which will gather samples from Phobos and closely study both moons, we are instead proposing that NASA guarantee a robust Participating Scientist Program for MMX: such programs have been strongly endorsed by SBAG and the other AGs. US-based scientists have strong expertise in the gathering and analysis of data from the types of spectrometers and other instruments on the MMX payload.

\section{Discovery Science Goals}

There are no currently high priority Discovery class missions to small satellites. If IVO is selected, a flyby of an outer moon is encouraged. IVO's eccentric orbits offer plenty of time for dynamical and physical studies of the remote observations of the Jovian irregulars.

\section{- Recommendations on the optimum balance among small, medium, and large missions and research programs.}


Because our recommendations involve "ridealong" and serendipitous measurements, as well as a robust Participating Scientists Program for MMX, we have no specific recommendations. In general, there should be more Discovery missions because they are faster, can cover more targets, and are less expensive.

A robust research program on large telescopes, both Earth-based and orbital, is necessary to advance small satellite science. Current laboratory studies are piecemeal, a consequence of funding serendipitous proposals from scientists with a somewhat narrow focus. To provide a basis to prepare for and interpret data from missions, NASA needs a comprehensive set of spectra over a range of temperatures, grain sizes, geometry, and wavelengths and to that end should establish a facility with such an open-ended goal.

\section{- Recommendations on the balance among small, medium, and large missions and research programs when choices must be made across categories of programs because of limited funding.}

Flagship missions should not gobble up smaller missions, as the latter represent more goals, opportunities (especially for early-career scientists), and targets. Small missions also mean a faster turnaround, which enables building upon more recent technology. Instead the Flagships should be delayed or descoped to the "science floor". Missions should never eliminate or tax research programs, as basic research is the fountainhead of new mission concepts.

- What should be done if cost overruns begin to expand a mission budget to the point where other programs will be affected (negatively).

When costs are overrun, missions should be descoped by taking actions such as deleting instruments and putting members of the science team "on ice." Each mission proposal should include a "science floor" so missions can be descoped. Delays should be avoided, as they end up costing more in the long run and disrupt other programs (although an AO can be delayed). Missions should never be canceled, because taken together they represent a carefully thought plan of priorities by NASA.

\section{- How could/should new discoveries affect your priorities?}

Undoubtedly new satellites will be discovered, but they will not affect priorities because they are not driving missions. The goals relating to irregular satellites for Flagship and New Frontier Missions are pretty generic. Our strong endorsement of a Participating Scientist Program for MMX will not change.

\section{REFERENCES}

Ball, A, J. et al. 2009 Mars Phobos and Deimos Survey (M-PADS) - A Martian Moons orbiter and Phobos lander. Adv. In Space Res 43, 120-127.

Baumgärtel K., K. Sauer, A. Bogdanov, E. Dubinin and M. Dougherty 1996. "Phobos events": Signatures of solar wind dust interaction. Planet. Space Sci. 44, 589-601. 
Bottke, W. F., D. Nesvorny, D. Vokrouhlicky, and A. Morbidelli. 2010. The Irregular Satellites: The Most Collisionally Evolved Populations in the Solar System. A. J. 139, 994.

Britt, D. T and the Gulliver Team 2003. The Gulliver Mission: Sample return from the Martian moon Deimos. LPSC XXIV \# 1841.

Buratti, B J., M. Hicks, and A. Davies 2005. Spectrophotometry of the small satellites of and their relationship to Iapetus, Phoebe, and Hyperion. Icarus 175, 490-495.

Buratti, B. J. et al. 2019. Close Cassini Flybys of Saturn's Ring Moons Pan, Daphnis, Atlas, Pandora, and Epimetheus. Science 364, 1053.

Canup, R., E. Asphaug 2001. Origin of the Moon in a giant impact near the end of the Earth's formation. Nature 412, 708-712.

Cartright, R. et al. 2018. Red material on the large moons of Uranus: Dust from the irregular satellites? Icarus 314 210-231.

Denk, T. and Mottola, S. 2019. Studies of irregular satellites: I. Lightcurves and rotation periods of 25 Saturnian moons from Cassini observations. Icarus 322, 80-102.

Dubinin E. M., et al. 1990. Indirect evidences for a gas/dust torus along the Phobos orbit. GRL $17,861-864$

Johnson, T. V., J. Lunine, 2005. Saturn's moon Phoebe as a captured body from the outer Solar System System. Nature 435, 69-71.

Gladman, B. 2007. Origin and Evolution of Phobos and Deimos. First International Con. On Evolution of Phobos and Deimos. Moffett Fld, CA Nov. 5-8, 2007, \# 1377.

Jewitt, D., N. Haghighipour 2007. Irregular Satellites of the Planets: Products of Capture in the Early Solar System. ARA\&A 45, 262-295.

Morbidelli, A., H. F. Levison, K. Tsiganis, G. Gomes 2005. Chaotic capture of Jupiter's Trojan asteroids in the early Solar System, Nature 435, 462-465.

Nesvorny, D., D. Vokrouhlicky, A. Morbidelli 2007. Capture of Irregular Satellites during Planetary Encounters A. J. 133, 1962-1976.

Nesvorný, D. et al. 2014. Capture of Irregular Satellites at Jupiter. Ap. J. 784, 22. doi:10.1088/0004-637X/784/1/22

Nicholson, P., M. Cuk, S. S. Sheppard, D. Nesvorny, T. V. Johnson 2008. The Solar System beyond Neptune, 411-424.

Pieters, C. et al. 2009. Aladdin : Exploration and sample return from the Moons of Mars: Concepts and approaches for exploration. http://www.lpi.usra.edu/meetings/robomars/pdf/6093.pdf

Showalter, M. R.; D. P. Hamilton, P. D. Nicholson 2006. A deep search for Martian dust rings and inner moons using the Hubble Space Telescope, Planet. Space Sci. 54, 844-854.

Soter S., 1971. The dust belts of Mars. Report of Center for Radiophysics and Space Res. 462.

Stern et al. 2019. Initial results from the New Horizons exploration of 2014 MU69, a small Kuiper Belt object. Science 364. DOI: 10.1126/science.aaw9771.

Thomas, P., Helfenstein, P. 2020. The small inner satellites of Saturn: Shapes, structures and some implications. Icarus 144.

Tiscareno M.et al. 2019. Close-range remote sensing of Saturn's rings during Cassini's ringgrazing orbits and Grand Finale. Science, Volume 364, Issue 6445, id. aau1017 (2019).

Tsiganis, K., G. Gomes, A. Morbidelli, H. F. Levison 2005. Origin of the orbital architecture of the giant planets of the Solar System. Nature 435, 459-461.

Weaver, H. et al. 2016. The small satellites of Pluto as observed by New Horizons. Science 351. Issue 6279 , id.aae 0030 